\title{
Familial influence on variation in age of onset and behavioural phenotype in Alzheimer's disease
} NIGEL TUNSTALL, MICHAEL J. OWEN, JULIE WILLIAMS, FRANCES RICE,
STEPHANIE CARTY, SARA LILLYSTONE, LINDSAY FRASER, PATRICK KEHOE,
DAVID NEILL, VARUNI RUDRASINGHAM, PAK SHAM and SIMON LOVESTONE

\section{Background Alzheimer's disease} manifests considerable heterogeneity, the cause of which is unknown.

\begin{abstract}
Aims To determine the familial (genotypic) influence on phenomenology (phenotype) in Alzheimer's disease.
\end{abstract}

\begin{abstract}
Method Affected sibling pairs with Alzheimer's disease were assessed for a range of cognitive and non-cognitive symptoms. Resemblance for phenotypic characteristics was estimated using intraclass correlations for continuous traits and by pairwise concordance for dichotomous traits. The relationship between age of onset and APOE genotype was examined using linear regression analysis.
\end{abstract}

\section{Results Significant familial effects on} age of onset (intraclass correlation 0.4I) and mood state (intraclass correlation 0.26), and a relatively high pairwise concordance for agitation (excess concordance 0.1 ) were found. The APOE locus was found to account for $4 \%$ of the variance in age of onset.

\section{Conclusions Substantial familial influence on age of onset, depression and agitation suggests that genotype does influence phenotype in Alzheimer's disease. Establishing the molecular basis for this phenotypic variation may prove relevant to other neuropsychiatric disorders.}

\section{Declaration of interest Funding received from the Medical Research Council (project grant G9530757N).}

The reasons for the considerable clinical heterogeneity of Alzheimer's disease are unknown; we hypothesised that it might reflect genetic heterogeneity. Families with autosomal dominant Alzheimer's disease have different ages of onset (Mullan et al, 1993), and in late-onset Alzheimer's disease variation in the apolipoprotein $\mathrm{E}$ gene $(A P O E)$ is associated with variation in onset age (Meyer et al, 1998). Aphasia and apraxia have been found to be associated with familial Alzheimer's disease (Breitner \& Folstein, 1984; Lampe et al, 1994). Depressive symptomatology characterises some families with early-onset Alzheimer's disease (Harvey et al, 1998), and a family has been reported in which Alzheimer's disease was associated with a schizophrenialike psychosis (Sumi et al, 1992). For lateonset Alzheimer's disease, genes have been associated with psychotic symptoms in Alzheimer's disease (Holmes et al, 1998; Sweet et al, 1998). However, no systematic study of familial resemblance for phenotypic traits has been reported previously. We therefore conducted an affected sibling-pair phenotype study.

\section{METHOD}

\section{Sample}

As part of a molecular genetic study of Alzheimer's disease, DNA samples and clinical data were collected on affected sibling pairs with Alzheimer's disease, ethical permission having been obtained from the Multicentre Research Ethics Committee and relevant local ethics committees. The sample consisted of White Europeans identified by old age psychiatrists in the UK and assessed by a psychiatrist (N.T.) or psychologist (F.R., S.C., S.L.). Before commencing the study, the psychiatrist and a psychologist assessed patients with Alzheimer's disease separately, and then the case was reviewed by a panel including an experienced clinician (S.L.). Training was conducted until all investigators reported identical assessment. Consent/assent was established for each individual and agreement was obtained from relatives and carers. Clinical history and current clinical state were established in a standardised manner for each individual; subjects meeting NINCDS-ADRDA criteria (McKhann et al, 1984) for a diagnosis of probable or possible Alzheimer's disease were included.

\section{Clinical assessment}

The standardised interview schedule consisted largely of informant-based scales and batteries with established reliability; of relevance to the present report are: the CAMDEX (Roth et al, 1986), from which age of onset data were obtained; the Cornell Scale for Depression in Dementia (CSDD; Alexopoulos et al, 1988); and the Manchester and Oxford Universities Scale for the Psychopathological Assessment of Dementia (MOUSEPAD; Allen et al, 1996). The CSDD is a clinician-administered instrument that utilises information from interviews with both the patient and an informant. Total CSDD scores correlate highly with depressive subtypes classified according to Research Diagnostic Criteria (RDC). The MOUSEPAD is a 59-item instrument that measures psychopathology and behavioural changes in dementia; it has established reliability, sensitivity and validity (Allen et al, 1996).

\section{APOE genotyping}

Deoxyribonucleic acid was extracted from venous blood and APOE genotype was determined using standard methods (Wenham et al, 1991).

\section{Data analysis}

Phenotypic data relating to age of onset and behavioural and psychiatric signs and symptoms of dementia were analysed using the Statistical Package for the Social Sciences. The behavioural variables were treated as separate from one another. Support for this approach is given by a factor analysis of behavioural changes in dementia that identified three robust syndromes overactivity, aggressive behaviour and psychosis - that were stable across time (Hope et al, 1997). Variability in continuous phenotypic traits was partitioned into betweenand within-family variation using analysis of variance, in order to distinguish familial from non-familial sources of variation; 
intraclass correlations were calculated to quantify the proportion of trait variance accounted for by familial effects, and hence to estimate the strength of familial influences on these traits. Because case-finding for this sample involved recruiting families with two or more living affected siblings, it necessarily excluded deceased affected siblings and siblings potentially at risk but currently below the age range of susceptibility for the disorder. However, age of onset data on deceased affected siblings were included where obtainable. For all other data points, only those clinical variables directly assessed with a living subject were utilised. The relationship between age of onset and APOE alleles was examined using linear regression analysis.

For each dichotomous trait, a pairwise concordance for its presence (the number of pairs in which both siblings are positive for the trait of interest, divided by the total number of pairs) was calculated, as was the expected value under the null hypothesis that familial effects are not operating (calculated as the square of the prevalence of the trait in question).

\section{RESULTS}

\section{Age of onset}

A total of 106 affected sibling pairs with Alzheimer's disease were collected: $66 \%$ of subjects were female and $43 \%$ were living in an institutional setting at the time of sampling. Age of onset data were obtained on 76 pairs, 19 trios and two quartets from the 106 sibships, including those relating to 10 deceased siblings. Intraclass correlations were calculated for each of these three groups following appropriate weighting for sibship size, and a combined estimate, with upper and lower $95 \%$ confidence limits, was obtained using Fisher's $z$ transformation as described in the Appendix. Age of onset ranged from 46 to 91 years $($ mean $=73.61$ years, s.d. $=7.89)$. The intraclass correlation $(r)$ for familial influence on variation in age of onset was 0.41 $(95 \% \mathrm{CI}=0.25-0.62)$, indicating that approximately $40 \%$ of the variance in this trait is due to familial (genetic and/or shared environmental) effects. APOE genotype data were obtained on 101 sibships; the $\varepsilon 2, \varepsilon 3$ and $\varepsilon 4$ allele frequencies were $0.04,0.52$ and 0.44 , respectively. The $\varepsilon 4$ frequency is high in comparison to samples not restricted to multiplex pedigrees (a metaanalysis by Farrer et al (1997) produced a frequency estimate of 0.37 for Caucasians with Alzheimer's disease) and is consistent with our strategy of recruiting affected sibling pairs (or larger affected sibships), which are likely to be enriched for susceptibility genes for Alzheimer's disease. The regression coefficient for age of onset by APOE $\varepsilon 4$ gene dosage was $-1.73(95 \%$ $\mathrm{CI}=-4.77$ to 1.31 ), and by $A P O E \varepsilon 2$ gene dosage was $2.06(95 \% \mathrm{CI}=-5.37$ to 9.49$)$, consistent with previous reports that $\varepsilon 4$ lowers and $\varepsilon 2$ elevates the age of onset in a dose-dependent fashion. However, the effect of APOE explained only $4 \%$ of the variance in age of onset $(95 \% \mathrm{CI}=0.005$ 0.109 ). When subjects were dichotomised into groups based on the possession or non-possession of the $\varepsilon 4$ allele, the 140 subjects identified as having an $\varepsilon 4$ allele were calculated to have a mean age of onset of 73.26 years, and the 68 subjects identified as having no allele $\varepsilon 4$ were calculated to have a mean age of onset of 76.37 years: a between-group difference of 3.10 years ( $t$-test for equality of means: $t=2.61$, $P=0.01$, assuming unequal variances).

\section{Current mood state}

Data on current mood state (measured using position on the Cornell scale) was obtained on 86 pairs. The intraclass correlation for familial influence on variation in this dimension was $0.26(P<0.05)$.

\section{Dichotomous traits}

For the dichotomous measures of behavioural disturbance at some point during the clinical course, data were obtained on 99 pairs. As in previous studies, we found a high rate of behavioural disturbance, with frequencies as follows: agitation, 0.44; aggression, 0.44; psychosis, 0.41 (with delusions and hallucinations showing frequencies of 0.36 and 0.30 , respectively). The pairwise concordance for each trait (with the excess (observed-expected) concordance in parentheses) was as follows: agitation, 0.29 (0.1); aggression, 0.22 (0.03); psychosis, 0.21 (0.04).

\section{DISCUSSION}

\section{Familial influence on age of onset}

The study provides strong support for the possible role of genetic factors in determining age of onset, accounting for between a quarter and two-thirds of the variance of this trait. This is consistent with the results of a study (Alafuzoff et al, 1994) showing a greater proportion of early age of onset in multiplex, compared with simplex, families. The result obtained is necessarily an overestimate of the true correlation because siblings at risk but, as yet, unaffected were excluded from the sample. A truer estimate might be obtained in the future by a study employing a population-based casefinding design with complete ascertainment. Nonetheless, the present study goes beyond previous work in providing a quantitative estimate of familial effects on variation in this trait. It further suggests that genes other than $A P O E$ are likely to contribute to variation in age of onset - APOE is estimated to account for between 0.5 and $11 \%$ of the variance in age at onset of Alzheimer's disease, which is comparable to a previous estimate of $10 \%$ for dementia not restricted to Alzheimer's disease (Slooter et al, 1998). It has been argued that the role of the APOE $\varepsilon 4$ allele in conferring susceptibility to Alzheimer's disease may be mediated exclusively through modifying the age of onset; our data are compatible with a model in which other genes (either independently or in conjunction with $A P O E$ \&4) also modify the age of onset rather than conferring susceptibility per se.

\section{Familial influence on mood state}

Evidence of significant familial effects on current mood state within Alzheimer's disease has been detected. This finding is of considerable interest because the overrepresentation of depression in Alzheimer's disease has not hitherto been adequately explained. Although aetiological models suggest that genetic susceptibility and recent life stressors are the major determinants of depression in adults, behavioural genetic work points to genetic influence on variation in depressive symptoms as being greater in older, compared with younger, nondemented individuals (Gatz et al, 1992). $A P O E$ is probably a risk factor for neither late-life depression nor depression within Alzheimer's disease (Cantillon et al, 1997; Schmand et al, 1998). However, an association between depression in Alzheimer's disease and family history of affective disorder has been reported (Pearlson et al, 1990; Lyketsos et al, 1996; Strauss \& Ogrocki, 1996) suggesting the influence of genetic factors unrelated to susceptibility for Alzheimer's disease. 


\section{Familial influence on behavioural disturbance}

The finding of a significantly elevated pairwise concordance for agitation suggests moderate genetic influence on this trait. Evidence points to relative dopaminergic overactivity as a basis for agitation in $\mathrm{Alz}$ heimer's disease (Sweet et al, 1997). Candidates genes for propensity to agitation in Alzheimer's disease, therefore, include those genes influencing the functioning of this system, such as the dopamine receptors, polymorphisms in which have been reported to be associated with aggression in Alzheimer's disease (Sweet et al, 1998). In the present study, only a very modest excess concordance for the presence of aggression was detected. The interrelationship between agitation, aggression and dopamine receptor variation warrants further study.

\section{Genotype-phenotype correlation in Alzheimer's disease may identify 'psychiatric' genes}

The rich phenotypic variability of Alzheimer's disease cannot be explained exclusively by genetic variation but, nonetheless, this study does demonstrate that familial factors exert significant influence upon the pattern of symptoms experienced by individuals. Given that siblings are generally exposed to very little shared environment in later life, it seems very unlikely that shared environmental factors influence familial aggregation of symptoms in Alzheimer's disease. It will be important to replicate this finding in other studies and to examine different traits. Because phenotypic characteristics in Alzheimer's disease are not stable over time, it will be important to study larger sample sets over time.

Determining the genes that contribute to phenotypic variability in Alzheimer's disease will be important in understanding this disorder more fully. Behavioural disturbance is a major determinant of carer stress and an important target for therapy, and it is possible that better understanding of the underlying pathogenesis of behavioural disturbance may lead to improved clinical management. However, these findings may also have implications for other psychiatric genetic studies. One hypothesis following from our findings is that genetic vulnerability to psychiatric disorder is revealed by neurodegeneration. That is, some individuals may have a genetic propensity to depression, for example, but other factors protect against its development until

\section{CLINICAL IMPLICATIONS}

- Behavioural disturbance is common in Alzheimer's disease and may aggregate in families.

- Genetic factors increasing risk of psychiatric disorders, including depression, may be 'revealed' by Alzheimer's disease.

- Families multiply affected by Alzheimer's disease show resemblance for age of onset.

\section{LIMITATIONS}

- The estimate of familial influence on age of onset is necessarily an overestimate because at-risk siblings currently below the age of risk are excluded from the sample.

- Although the largest study of its kind, only 106 sibships were studied.

- The severity of behavioural disturbance was not assessed.

NIGEL TUNSTALL, MRCPsych, LINDSAY FRASER, BSc, SIMON LOVESTONE, PhD, Department of Psychiatry Institute of Psychiatry, London; MICHAEL J. OWEN, PhD, JULIE WILLIAMS, PhD, FRANCES RICE, BA, STEPHANIE CARTY, BSC, SARA LILLYSTONE, BSC, PATRICK KEHOE, PhD, VARUNI RUDRASINGHAM, BSC, Department of Psychological Medicine, University of Wales College of Medicine, Cardiff; DAVID NEILL, MRCPsych, Institute for Health of the Elderly, Newcastle, UK; PAK SHAM, MRCPsych, Department of Psychological Medicine, Institute of Psychiatry, London, UK

Correspondence: Dr N. Tunstall, Department of Psychiatry, Institute of Psychiatry, London SE5 8AF

(First received 29 March 1999, final revision 26 July 1999, accepted 27 July 1999)

the onset of Alzheimer's disease lowers the susceptibility threshold of these individuals. Our study suggests that in the search for genes for depression, and possibly other psychiatric disorders, Alzheimer's disease may prove a more fertile hunting ground.

\section{APPENDIX: ESTIMATION OF INTRACLASS CORRELATION FROM SIBSHIPS}

The data consist of 76 sibling pairs, 19 trios and two quartets. Intraclass correlations ( $r$ ) are estimated for these three subsets of data in three separate analyses of variance (ANOVA). These three estimates are then averaged to give an overall intraclass correlation, using the inverse of sampling variances as weights. For each type of sibship, all possible sibling pairs are formed (i.e. one, three and six sibling pairs for sibships of size two, three and four, respectively). These three sets of sibling pair data are subjected to ANOVA, to obtain three estimates of $r$ (calculated as $(M S B-M S W) /(M S B+M S W)$, where MSB and MSW are between- and within-pairs mean squares, respectively). These estimates of $r$ are subjected to Fisher's z-transformation, with the resulting normal variates having an approximate sampling variance of
$1 /(n-2)$, where $n$ is the number of pairs (Donner, 1986). Each sibling pair contributes one independent pair, each trio contributes two independent pairs and each quartet contributes three independent pairs. The numbers of independent pairs from sibling pairs, trios and quartets are therefore 76,38 and 6 , respectively. The normal variates $z$ are averaged according to the inverse of the sampling variances, and back-transformed to give an overall estimate of the intraclass correlation.

\section{REFERENCES}

Alafuzoff, I., Almqvist, E., Adolfsson, R., et al (1994) A comparison of multiplex and simplex families with Alzheimer's disease/senile dementia of Alzheimer's type within a well defined population. Journal of Neural Transmission, Parkinson's Disease and Dementia, 7, 61-72.

Alexopoulos, G. S., Abrams, R. C., Young, R. C., et al (1988) Cornell Scale for Depression in Dementia. Biological Psychiatry, 23, 27I-284.

Allen, N. H. P., Gordon, S., Hope, T., et al (1996) Manchester and Oxford Universities Scale for the Psychopathological Assessment of Dementia (MOUSEPAD). British Journal of Psychiatry, 169, 293-307.

Breitner, J. C. \& Folstein, M. F. (1984) Familial Alzheimer Dementia: a prevalent disorder with specific clinical features. Psychological Medicine, 14, 63-80. 
Cantillon, M., Harwood, D., Barker, W., et al (1997) No association between apolipoprotein E genotype and late-onset depression in Alzheimer's disease. Biological Psychiatry, 4I, 246-248.

Donner, A. (1986) A review of inference procedures for the intraclass correlation coefficient in the one-way random effects model. International Statistical Review, $\mathbf{5 4}$ 67-82.

Farrer, L. A., Cupples, L. A., Haines, J. L., et al (1997) Effects of age, sex, and ethnicity on the association between apolipoprotein $\mathrm{E}$ genotype and Alzheimer disease. A meta-analysis. APOE and Alzheimer Disease Meta Analysis Consortium [see comments]. Journal of the American Medical Association, 278, 1349-1356

Gatz, M., Pedersen, N. L., Plomin, R., et al (1992) Importance of shared genes and shared environments for symptoms of depression in older adults. Journal of Abnormal Psychology, 10I, 701-708.

Harvey, R., Ellison, D., Hardy, J., et al (1998)

Chromosome 14 familial Alzheimer's disease: the clinical and neuropathological characteristics of a family with a leucine-> serine (L250S) substitution at codon 250 of the presenilin I gene. Journal of Neurology, Neurosurgery and Psychiatry, 64, 44-49.

Holmes, C., Arranz, M. J., Powell, J. F., et al (1998) 5 $\mathrm{HT}_{2 \mathrm{~A}}$ and $5-\mathrm{HT}_{2 \mathrm{C}}$ receptor polymorphisms and psychopathology in late onset Alzheimer's disease. Human Molecular Genetics, 7, 1507-1509.

Hope, T., Keene, J., Fairburn, C., et al (1997)

Behaviour changes in dementia 2: Are there behavioural syndromes? International Journal of Geriatric Psychiatry 12, 1074-1078.

Lampe, T. H., Bird, T. D., Nochlin, D., et al (1994) Phenotype of chromosome 14-linked familial Alzheimer's disease in a large kindred. Annals of Neurology, 36, 368-378.

Lyketsos, C. G., Tune, L. E., Pearlson, G., et al (1996) Major depression in Alzheimer's disease. An interaction between gender and family history. Psychosomatics, 37, 380-384.

McKhann, G., Dracman, D., Folstein, M., et al (1984) Clinical diagnosis of Alzheimer's disease: report of the NINCDS-ADRDA Work Group under the auspices of the Department of Health and Human Services Task Force on Alzheimer's disease. Neurology, 34, 939-944.

Meyer, M. R., Tschanz, J.T., Norton, M. C., et a (1998) APOE genotype predicts when - not whether - one is predisposed to develop Alzheimer's disease. Nature Genetics, 19, 321-322

Mullan, M., Houlden, H., Crawford, F., et al (1993) Age of onset in familial early onset Alzheimer's disease correlates with genetic aetiology. American journal of Medical Genetics, 48, 129-130.

Pearlson, G. D., Ross, C. A., Lohr, W. D., et al (1990) Association between family history of affective disorder and the depressive syndrome of Alzheimer's disease. American Journal of Psychiatry, 147, 452-456.

Roth, M., Tym, E., Mountjoy, C. Q., Huppert, F. A., et al (1986) CAMDEX. A standardised instrument for the diagnosis of mental disorder in the elderly with special reference to the early detection of dementia. British Journal of Psychiatry, 149, 698-709.

Schmand, B., Hooijer, C., Jonker, C., et al (1998) Apolipoprotein E phenotype is not related to late-life depression in a population-based sample. Social Psychiatry and Epidemiology, 33, 21-26.

Slooter, A. J., Cruts, M., Kalmijn, S., et al (1998) Risk estimates of dementia by apolipoprotein E genotypes from a population-based incidence study: The Rotterdam Study. Archives of Neurology, 55, 964-968.

Strauss, M. E. \& Ogrocki, P. K. (1996) Confirmation of an association between family history of affective disorder and the depressive syndrome in Alzheimer's disease. American journal of Psychiatry, 153, 1340-1342.

Sumi, S. M., Bird, T. D., Nochlin, D., et al (1992) Familial presenile dementia with psychosis associated with cortical neurofibrillary tangles and degeneration of the amygdala. Neurology, 42, 120-127.

Sweet, R., Pollock, B. G., Mulsant, B., et al (1997) Association of plasma homovanillic acid with behavioural symptoms in patients diagnosed with dementia: a preliminary report. Biological Psychiatry, 42, 1016-1023.

_, Nimgaonkar, V. L., Kamboh, M. I., et al (1998) Dopamine receptor genetic variation, psychosis, and aggression in Alzheimer's disease. Archives of Neurology, 55, 1335-1340.

Wenham, P. R., Price, W. H. \& Blandell, G. (1991) Apolipoprotein E genotyping by one-stage PCR. Lancet, 337, $1158-1159$ 